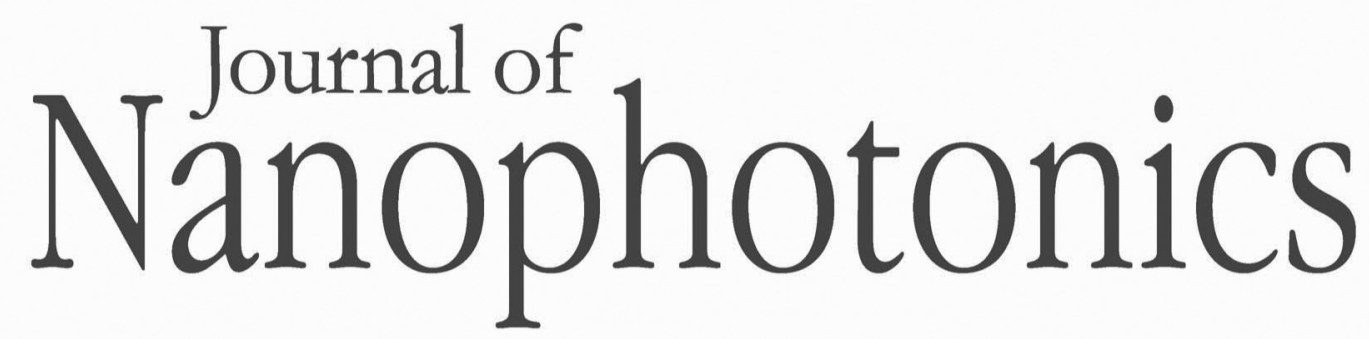

\title{
Review of a viral peptide nanosystem for intracellular delivery
}

\author{
Annarita Falanga \\ Rossella Tarallo \\ Emilia Galdiero \\ Marco Cantisani \\ Massimiliano Galdiero \\ Stefania Galdiero
}




\title{
Review of a viral peptide nanosystem for intracellular delivery
}

\author{
Annarita Falanga, ${ }^{a}$ Rossella Tarallo, ${ }^{\text {a }}$ Emilia Galdiero, ${ }^{\text {b }}$ Marco Cantisani, \\ Massimiliano Galdiero, ${ }^{c}$ and Stefania Galdiero ${ }^{a}$ \\ ${ }^{a}$ University of Naples Federico II, Department of Biological Sciences, Division of Biostructures \\ and Centro Interuniversitario di Ricerca sui Peptidi Bioattivi, Via Mezzocannone 16, \\ 80134, Napoli, Italy \\ sgaldier@unina.it \\ ${ }^{b}$ University of Naples Federico II, Department of Structural and Functional Biology, \\ Viale Cinzia, Monte Sant'Angelo, Napoli, Italy \\ ${ }^{c}$ II University of Naples, Department of Experimental Medicine, Via De Crecchio 7, \\ 80138, Napoli, Italy
}

\begin{abstract}
The internalization of bioactive molecules is one of the most critical problems to overcome in theranostics. In order to improve pharmacokinetic and pharmacodynamic properties, synthetic transporters are widely investigated. A new nanotechnological transporter, gH625, is based on a viral peptide sequence derived from the herpes simplex virus type 1 glycoprotein $\mathrm{H}(\mathrm{gH})$ that has proved to be a useful delivery vehicle, due to its intrinsic properties of inducing membrane perturbation. The peptide functionalization with several kinds of nanoparticles like quantum dots, dendrimers, and liposomes could be of particular interest in biomedical applications to improve drug release within cells, to increase site-specific action, and eventually to reduce related cytotoxicity. (C) The Authors. Published by SPIE under a Creative Commons Attribution 3.0 Unported License. Distribution or reproduction of this work in whole or in part requires full attribution of the original publication, including its DOI. [DOI: 10.1117/1.JNP.7.071599]
\end{abstract}

Keywords: quantum dots; peptide; virus; delivery; dendrimer; liposomes.

Paper 12136V received Oct. 3, 2012; revised manuscript received Dec. 13, 2012; accepted for publication Dec. 14, 2012; published online Jan. 16, 2013.

\section{Introduction}

The membrane bilayer is a semipermeable barrier defining the interior (or cytoplasm) of an individual cell. The existence of this finely tuned barrier confers to cells their potential to survive and function properly. Nevertheless, crossing the cellular membranes remains a major obstacle for the delivery of therapeutics. ${ }^{1,2}$ The lipophilic nature of biological membranes restricts the direct intracellular delivery of most compounds, and whereas small molecules and ions can diffuse across the barrier, large molecules (more than $1 \mathrm{kD}$ ) are generally excluded from simple diffusion into the cell. The differing hydrophobicity/hydrophilicity of the lipid membrane can make transfer across this barrier extremely difficult, due to differences in solubility.

Novel compounds with therapeutic potential are strictly dependent on their pharmacodistribution properties to reach the stage of pharmaceutical preparations and stimulate industrial interest. In particular, many pharmaceutical agents should be delivered intracellularly to exert their therapeutic action inside the cytoplasm or on individual organelles, such as nuclei (targets for gene and antisense therapy), lysosomes (targets for the delivery of deficient lysosomal enzymes) and mitochondria (targets for pro-apoptotic anticancer drugs).

Several basic cellular mechanisms can be exploited for the intracellular delivery of a compound across the plasma membrane. There are active mechanisms, such as endocytosis, and passive mechanisms, such as translocation across the lipid bilayer; alternatively, there are highly invasive procedures, such as microinjection and/or electroporation, which could cause transient damage to membranes. The use of viral particles as delivery tools, which seemed very promising for gene therapies, presents several problems in terms of side effects and toxicity. ${ }^{3}$ 
Recently, cell-penetrating peptides (CPPs) have been used extensively, due to their capability to transport several kinds of macromolecules across the bilayer in vitro and in vivo. ${ }^{4-6}$

CPPs are short and usually basic amino acid rich peptides that can cross biological barriers. Although the mechanism of cellular uptake is still unknown, CPPs are able to deliver various entities into cells, including small molecules, peptides, proteins, DNA/RNA, liposomes, and other supramolecular aggregates. They are usually divided into three classes: protein derived peptides (such as TAT, penetratin or pANTP, transportan, and HSV-1 VP22), amphipathic peptides resulting from the sequential assembly of hydrophobic and hydrophilic domains (such as MAP, MPG, and Pep-1), and synthetic or cationic peptides such as poliarginines (such as Arg9). ${ }^{7}$

The uptake mechanism of CPPs is still debated and is believed to involve mainly the endocytic pathway, trapping the conjugated cargo in endosomes and eventually ending in lysosomes, where common enzymatic degradation mechanisms take place, decreasing its intracellular bioavailability. In order to avoid the endocytic pathway completely or partially, it is paramount to discover new molecules with different mechanism of uptake.

Recently, great attention has been devoted to viruses; in fact, many viruses have evolved quite efficient systems for endosomal release. ${ }^{8}$ Therefore, the discovery of new methodologies to reproduce their behavior represents a key objective. Since viruses may enter cells either through a endosomal pathway or via direct fusion on the plasma membrane through the activity of membranotropic peptides, great attention has been devoted to the study of hydrophobic peptides that traverse biological membranes efficiently, promoting lipid-membrane reorganizing processes such as fusion or pore formation and involving temporary membrane destabilization and subsequent reorganization, ${ }^{7,9}$ which may be able to circumvent the endosomal entrapment by favoring the escape from the endosome or by translocating a cargo through the plasma membrane directly into the cytosol. Delivery across cellular membranes involves several mechanisms, such as direct transfer through the cell surface membrane by lipid membrane fusion or transient permeabilization of the cell membrane. Alternatively, following endocytosis, transfer across vesicular membranes by lipid disruption, pore formation, or fusion may take place. Several of these membrane reorganization steps are also involved in the cell entry of viruses and other microorganisms, as well as being triggered by protein toxins and defense peptides. ${ }^{10}$ Several related processes, such as intracellular vesicle budding, cell-to-cell fusion, ${ }^{9}$ sperm-egg fusion, and the immune response, share common features with the mechanism of viral-induced membrane fusion.

The 19-residue peptide gH625 (from aa 625 to aa 644) is a membrane-perturbing domain derived from the $\mathrm{gH}$ fusion glycoprotein of herpes simplex virus type $\mathrm{I} \cdot{ }^{11,12}$ It interacts with biological membranes and is implicated in the merging of the viral envelope and the cellular membrane. ${ }^{13,14}$ The peptide contains particular residues that are crucial for its capacity to interact with and destabilize target lipid membranes. It is rich in hydrophobic residues, including glycines, leucines, alanines, and aromatic residues such as tryptophan and tyrosines, which are known to be located preferentially at the membrane interface. This hydrophobic domain is also crucial for insertion of the peptide into the membrane.

An amphipathic $\alpha$-helix is believed to be an important feature of fusion peptides, playing a crucial role in mediating lipid-protein interactions during the binding of proteins to membranes. Once bound, the hydrophobic face of the amphipathic peptide would allow the peptide to enter the membrane interior with an oblique orientation, thereby triggering local fusion of the membrane leaflets, pore formation, cracks, and membrane fusion.

The viral membranotropic peptide gH625 has been shown to interact strongly with and penetrate the lipid-phase spontaneously and insert into membranes. ${ }^{15}$ The peptide-lipid interactions are initiated by the arginine residue located at the C-terminus; in fact, when the arginine is mutated, the fusogenic activity of the peptide is strongly impaired. ${ }^{16}$ Compared with the TAT peptide, which mainly exploits the endocytic pathway, gH625 crosses membrane bilayers mainly through a translocation mechanism. A version of this fusogenic peptide one amino acid shorter than HSV-1 gH (missing the histidine at position 625) was also found to improve the endosomal release of DNA/Lipofectamine lipoplexes and transgene expression up to 30-fold in human cell lines. ${ }^{17}$ It has been recently demonstrated that gH625 can traverse the membrane bilayer and transport into the cytosol several compounds, such as quantum dots (QDs), ${ }^{16}$ liposomes, ${ }^{18}$ $\mathrm{NPs},{ }^{19}$ and dendrimers. ${ }^{20}$

This review focuses on different delivery applications of gH625. 


\section{Functionalization of Quantum Dots}

QDs are a new class of fluorescent probes under intense research and development for broad applications in molecular, cellular, and in vivo imaging; compared with organic dyes and fluorescent proteins, they have unique functional and structural properties, such as size and composition-tunable fluorescence emission, large absorption cross sections, and exceptional brightness and photostability. ${ }^{21}$ Furthermore, QDs are a well-characterized family of engineered nanostructures that provide an excellent model system to test toxicity. Many in vitro studies have suggested that nanomaterials almost always induce toxic responses, which is essentially correlated to the particle breakdown and subsequent release of toxic metal ions ${ }^{22,23}$ and the production of reactive oxygen species. ${ }^{24}$ However, properly coated and passivated QDs do not exhibit acute toxicity in vivo. ${ }^{25,26}$ Thus, the coating of QDs with peptide delivery molecules represents a novel strategy to enhance delivery and reduce toxicity.

In addition, the excellent photostability of QDs gives them great potential in cellular labeling and bioimaging, which have been exploited in different kinds of biological systems. ${ }^{21}$ Although considerable success has been achieved in using QDs for labeling fixed cells and for imaging cell membrane proteins, only limited progress has been made for molecular imaging inside living cells. Thus, wide application of QDs to intracellular and molecular imaging has been hampered by their insufficient ability to traverse cell membranes. Therefore, in order to use QDs as biological probes for intracellular applications, their delivery needs to be significantly improved, and the methods actually used are still inefficient. Several authors have recently reported on the functionalization of QDs with the TAT peptide or other positively charged CPPs as an enhancer of cell penetration, and they have established that the main route of entrance is via endosomal uptake; therefore, escape from the endosomal system is paramount. ${ }^{27-29}$ There is thus a great need to identify novel molecules that use different internalization mechanisms and may prove useful in the delivery of QDs. ${ }^{11}$

In order to assess the ability of gH625 to deliver a cargo inside the cell, QDs were used as a model. QDs alone have a poor affinity for the plasma membrane of HeLa cells at the concentration of $50 \mathrm{nM}$. The peptide conjugation of QDs enhanced their membrane-bound ability, providing a favorable condition for cellular internalization. Moreover, the gH625-QDs are internalized more effectively than TAT-QDs, and their internalization only partially involves the endocytic pathway. Furthermore, gH625 showed very low toxicity during in vitro studies when used at concentrations of up to $400 \mu \mathrm{M}$. Therefore, QDs conjugated with gH625 may represent a novel and useful delivery tool.

It is important that, when conjugated with QDs, gH625 exerts an action of translocation through cellular plasma membranes, which seems to be only relatively dependent on the endocytic route of entry. It has been previously reported that TAT peptides conjugated with small molecules enter cytoplasm and eventually the cell nuclei, while TAT QDs remain trapped in vesicles. This difference in the intracellular fate of TAT peptide-conjugated molecules and TAT QDs is likely to be a consequence of the difference in the size of the cargo. QDs have a diameter of about 1 to $10 \mathrm{~nm}$ and cannot escape efficiently from vesicles. On the contrary, gH625-QDs are present in the cytoplasm in a more punctuated form in comparison with TAT QDs. ${ }^{16}$ Therefore, a difference in mechanism of entry between TAT-QDs and gH625-QDs has been hypothesized, and this is of particular interest because peptides with different biophysical characteristics can condition the entry pathway of the same macromolecular cargo. These results provide new insights into the mechanism of gH625-mediated delivery and for the development of nanoparticle probes for intracellular targeting and imaging (Fig. 1). Similar delivery methods may enable the implementation of the next generation of QDs capable of long-term intracellular monitoring.

\section{Functionalization of Lipid-Based Nanocarriers}

Liposomal aggregates have been successfully used as in vivo carriers of active principles; ${ }^{30}$ they display some unique pharmacokinetic properties and can be adapted to a wide range of therapeutic agents. Liposomes are nontoxic, biodegradable, and non-immunogenic, and their size ranges in mean diameter from 50 to $300 \mathrm{~nm}$. The use of liposomal drugs could help in protecting the encapsulated drugs from chemical or metabolic degradation after injection, in reducing 


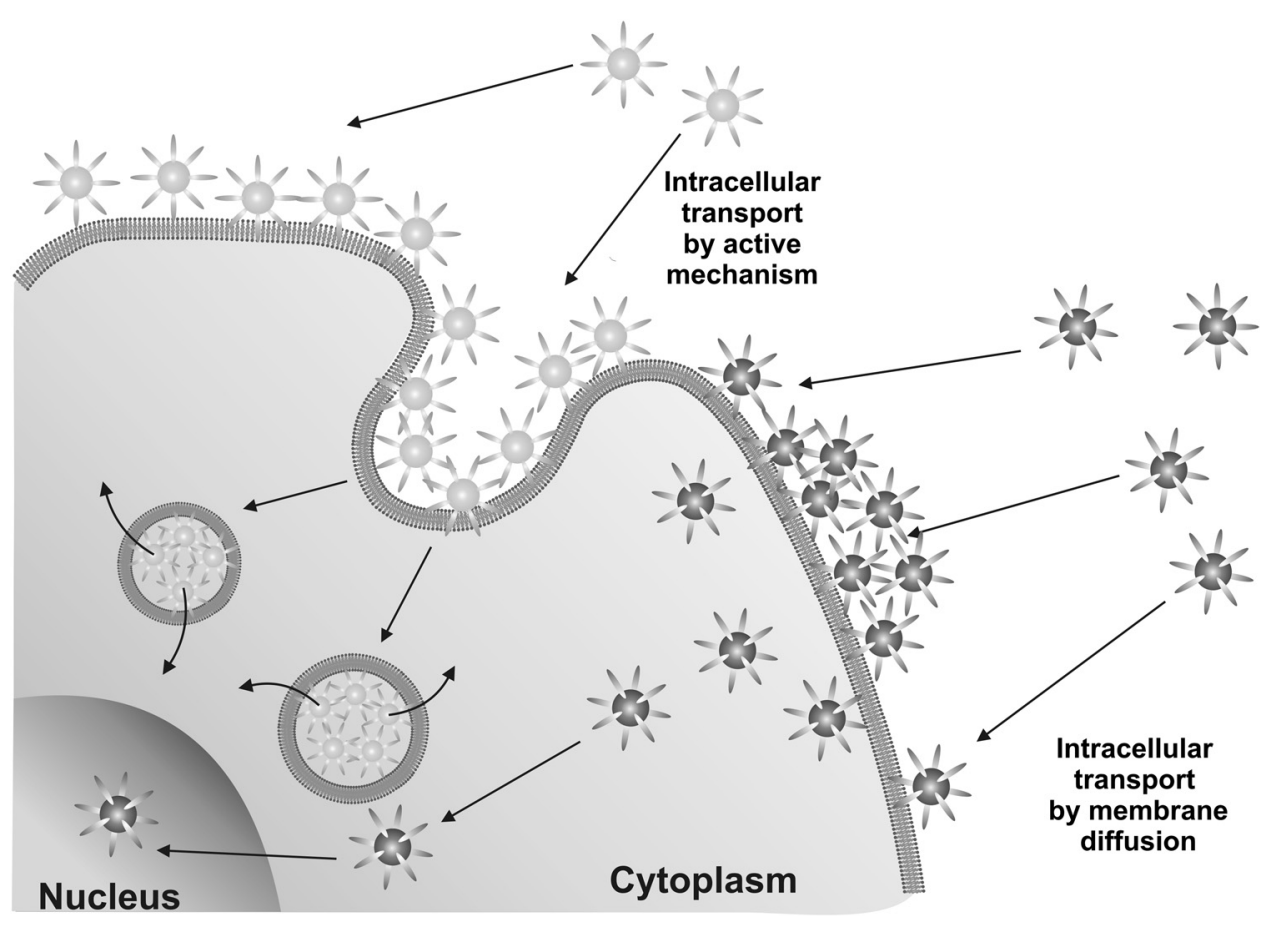

Fig. 1 Intracellular delivery: endocytic mechanisms compared to translocation.

toxicity through decreased exposure of anti-neoplastics to susceptible healthy tissues, and in enhancing antitumor activity resulting from a relatively long systemic circulation time, an extended exposure, and tumor-selective accumulation at specific sites of tumor growth. In particular, liposomes exhibit preferential extravasation and accumulation at the site of solid tumors, due to increased endothelial permeability and reduced lymphatic drainage in these tissues, which has been defined as enhanced permeability and retention effect. ${ }^{31} \mathrm{~A}$ drug encapsulated in liposomes shows marked changes in its pharmacokinetic and pharmacodynamic properties and lower systemic toxicity, while being also protected from early degradation and/or inactivation. ${ }^{32}$

To enhance the antitumor efficacy of liposomal drugs, many research groups are working toward the improvement of their cellular internalization through the addition of surface ligands.

Several peptides, such as penetratin and TAT, have been successfully used for the intracellular delivery of liposomes. ${ }^{33}$ Liposomes decorated with gH625 and loaded with doxorubicin can be prepared by different synthetic procedures which involve the coupling of the peptide to the lipid moiety before or after the assembly of the liposome. The ideal strategy depends strongly on the peptide and on the drug. The coupling of a hydrophobic peptide is complicated by the low solubility of the compound and by the higher tendency of the peptide to locate in the hydrophobic region of the bilayer, rather than on the external surface. Thus, the preferable way to obtain liposomes functionalized on the surface with a hydrophobic peptide consists of the coupling of peptide derivatives on pre-assembled liposomes. gH625-modified liposomes were prepared by using a post-aggregation strategy according to click-chemistry procedures. ${ }^{18}$ The ability of gH625-functionalized liposomes loaded with doxorubicin to penetrate inside cells was evaluated by confocal microscopy experiments.

Cells were incubated with a 1- $\mu \mathrm{m}$ solution of free Dox, of Dox-loaded liposomes, and of gH625-functionalized liposomes at $37^{\circ} \mathrm{C}$. The results obtained after five hours of incubation show that, as expected, free Dox can enter the cell and translocate into the nucleus. Dox-loaded liposomes also enter cell nuclei, where fluorescence due to Dox accumulation in DNA can be observed together with a slight diffuse fluorescence in the cytoplasm suggestive of Dox release from the liposomes. Conversely, gH625-functionalized liposomes encapsulating Dox accumulate in the cytoplasm without entering the nucleus. These results suggest that the functionalization of liposomes with gH625 could affect the uptake mechanism of liposomes, escaping the lysosome accumulation, allowing a more homogeneous intracytoplasmic distribution, and modulating the Dox release. Although the mechanism still remains to be established, the 
Falanga et al.: Review of a viral peptide nanosystem...

obtained results could be useful in the design of carriers for a controlled delivery and release of Dox in order to avoid side effects associated with Dox itself.

\section{Functionalization of Polymeric Nanocarriers}

Dendrimers are perfectly branched macromolecules with a well-defined structure ${ }^{34}$ that exhibit properties very different from linear polymers with the same composition and molecular weight. ${ }^{35}$ The high concentration of terminal functional groups on a dendrimer's surface determines the solubility of the entire compound-dendrimers have been used as unimolecular micelles, i.e., hydrophobic dendrimers that are water soluble due to peripheral hydrophilic groups or vice versa ${ }^{36,37}$ Traditional dendrimers are synthesized from monomers with the structure $\mathrm{AB}_{n},{ }^{38-40}$ from one generation to the following, the dendrimers grow radially with an exponential increase in both mass and number of free termini but only a minimal increase in the physical size of the structure. This causes the termini to become more closely packed. Unlike many other scaffolds, however, dendrimers have the benefit of a highly controlled synthesis, as well as yielding a single monodisperse compound, giving perfect control over the size, weight, and terminal functionalities of the resulting structure. Moreover, highly branched structures generally exhibit longer blood circulation times, due to their inability to pass through renal pores. ${ }^{41}$ Dendrimers have been shown to have extended lifetimes in vivo ${ }^{42}$ whereas lipid complexes are usually cleared from circulation rapidly by splenic and hepatic phagocytes. ${ }^{43}$

Dendrimers are now considered a very promising tool for drug delivery, thus combining the advantageous features of nanoparticles (such as ideal size as in vivo carriers and multivalency) of polymeric materials (such as low cost, tunable properties, and biocompatibility) and of small molecules (such as monodispersity and detailed control of their properties)..$^{35,44}$ The mechanism of dendrimer uptake and intracellular trafficking is yet to be established. ${ }^{45}$ PAMAM dendrimers ${ }^{46}$ and PAMAM dendrimers functionalized with the TAT peptide ${ }^{47}$ have been proved to involve essentially endocytosis mechanisms for internalization and intracellular trafficking, indicating that the addition of TAT failed to enhance delivery efficiency. Other studies using cationic PAMAM dendrimers showed that the dendrimers can insert themselves into the lipid bilayer, thanks to their potential to weaken the membrane bilayer or form holes. ${ }^{48}$

The combination of the benefits of dendrimers and peptide chemistry may prove useful for the development of a selective carrier that could cross the membrane and be efficiently targeted inside the cell. Targeted dendrimeric systems offer several advantages in their delivery, and their surface modification by means of conjugation or adsorption of a biospecific ligand may allow their delivery to specific sites and the modulation of drug release, minimizing toxic effects and increasing intracellular bioavability. ${ }^{49}$

The binding of gH625 to the dendrimer combines the benefits of dendrimer chemistry with those of a cell internalization unit that can cross the cell membrane mainly via a non-active translocation mechanism. The synthesis of a poly(amide)-based dendrimer containing terminal peptide chains and their cell uptake in vitro has been reported recently. The chosen dendrimers had been previously shown to exhibit high biocompatibility, due to their peptide-like backbone. $^{50,51}$ The structure of peptidodendrimer, the poly(amide) dendrimer terminated by gH625 peptides, is shown in Fig. 2. The peptidodendrimer can fuse model membranes like liposomes even more significantly than the peptide alone. Moreover, it is unable to fuse the inner monolayer or induce leakage of vesicles. The peptidodendrimer's ability to enter the cells was confirmed by using both qualitative and quantitative methods, and evidence showed that the peptidodendrimer is able to enter cells through a passive translocation mechanism, allowing the cargo to be released directly into the cytoplasm, as opposed to entrapment in endosomes, from which the cargo may not be able to escape before lysosomal degradation. Thus, the dendrimeric scaffolds may be a promising tool for an efficient drug delivery engine. ${ }^{20}$

\section{Functionalization of Polystyrene Nanoparticles}

Therapies for neurological disorders actually are limited by the inability of the therapeutic agents to cross the blood-brain barrier (BBB) efficiently. ${ }^{52}$ The BBB is a selective and dynamic barrier 


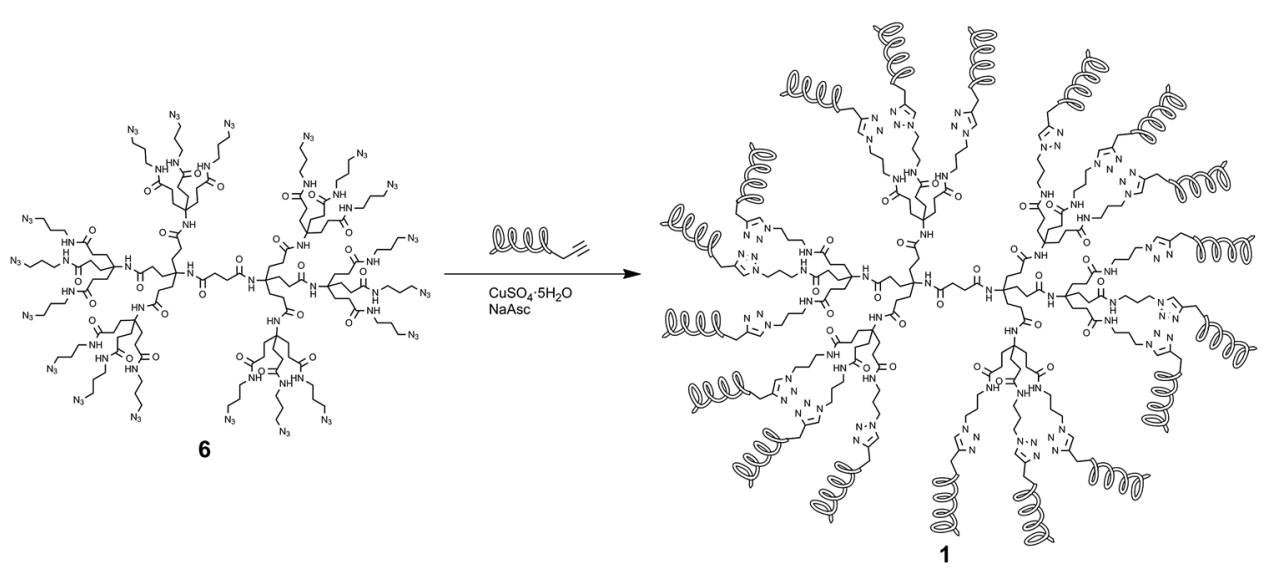

Fig. 2 Structure of peptidodendrimer. The helices represent the gH625 peptide sequence.

protecting the brain against invading organisms and unwanted substances; it also represents a formidable obstacle to the effective delivery of drugs to the central nervous system (CNS). For this reason, it is fundamental to identify and develop more effective strategies to enhance drug delivery to the brain for the therapy and diagnosis of neurological pathologies.

It is now well known that there are several invasive or non-invasive transport routes by which drugs enter the brain. The invasive approaches mainly consist of a temporary disruption of the $\mathrm{BBB}$, allowing the entry of a drug into the CNS, or of direct injection by means of intraventricular or intracerebral administration, ${ }^{53}$ while the non-invasive ones involve the systemic application of colloidal drug carriers undergoing a receptor or adsorptive mediated transcytosis mechanism ${ }^{54}$ or the passing of the BBB via intranasal delivery. ${ }^{55}$

To overcome this problem, fluorescent polystyrene nanoparticles could be functionalized with the membranotropic peptide gH625. Nanomaterials, such as nanoparticles, differ from other materials, due to a number of special characteristics, including small particle size, large surface area, shape, chemical composition, and charge. Together these characteristics give nanoparticles numerous advantages over other delivery systems, and the targeted delivery of drugs using nanocarriers for the treatment of diseases is a major focus of interest. ${ }^{56}$

The effect of gH625 on the penetration of 100-nm polystyrene nanoparticles (NPs) was analyzed using an in vitro BBB model based on bEnd3, an immortalized mouse cerebral endothelial cell line. The intracellular uptake of NPs with gH625 by brain endothelial cells was greater than that of the unfunctionalized NPs, and their intracytoplasmatic motion was mainly characterized by a random-walk behavior indicative of a passive mechanism of internalization. Most importantly, the gH625 peptide decreased NP intracellular accumulation as large aggregates and enhanced the NPs' crossing of the BBB.

This system represents a cell translocation motif never reported before in a BBB in vitro analysis and could be applied to the design of a drug delivery system homing to the brain and bypassing the endocytosis entrapment. Therefore, these novel nanoshuttles could be regarded as a promising strategy for engine delivery systems for the administration of therapeutical compounds to the brain. ${ }^{19}$

\section{Conclusions}

Cell-penetrating peptides are considerably important for the purpose of delivering cargoes to the insides of cells, and hundreds of sequences fall within CPP classification. The process by which membrane translocation is achieved has received great attention with the aim of raising the efficiency of penetration. Most well-studied peptides are able to deliver themselves as well as a cargo across the plasma membrane, although there is strong support for the involvement of endocytic pathways. The discovery of novel systems that use non-canonical mechanisms for internalization may lead to greater efficiency, both from a therapeutic and a diagnostic point of view. Most drugs are internalized by endocytosis mechanisms with a consequential set of limitations in biodistribution, specificity, and toxicity. On the contrary, novel delivery tools that are able to 
Falanga et al.: Review of a viral peptide nanosystem...

cross biological membranes using a completely or at least partially different mechanism of internalization may open up new avenues for the administration of drugs with a lower impact on the health of patients. This review focuses on recent data obtained on a viral membranotropic peptide gH625 derived from herpes simplex type 1 and its use, as well as the advantages over other CPPs as a drug delivery tool. The data summarized in this review suggest that membranotropic sequences represent a new and valuable tool for intracellular delivery for applications in theranostics. The opportunity to use a delivery tool with a different internalization mechanism may represent the possibility of reducing the dosages and thus increasing the results of therapies, contemporaneously reducing toxicity.

In conclusion, nanothechnology is developing rapidly and is addressing the safety issue correlated with the use of nanosystems as drug delivery systems, especially for long-term applications. The use of QDs is also particularly important, as they represent a versatile platform which combines unique physical, chemical, and optical properties that may facilitate in-depth studies of interactions of nanosystems with biological systems through real-time monitoring of biodistribution, intracellular uptake, drug release, and long-term fate. QDs offer a powerful platform for studying the behavior of a diverse set of nanovehicles, leading to the design of novel systems for drug delivery.

\section{Acknowledgments}

We thank MIUR for financial support (RBAP114AMK/005). We thank A. F. and M. C. for studies on QDs, R. T. for studies on dendrimers, and E. G. and M. G. for in vitro studies.

\section{References}

1. V. P. Torchilin, "Multifunctional nanocarriers," Adv. Drug Deliv. Rev. 58(14), 1532-1555 (2006), http://dx.doi.org/10.1016/j.addr.2006.09.009.

2. L. M. Bareford and P. W. Swaan, "Endocytic mechanisms for targeted drug delivery," Adv. Drug Deliv. Rev. 59(8), 748-758 (2007), http://dx.doi.org/10.1016/j.addr.2007.06 .008 .

3. J. K. Raty et al., "Improving safety of gene therapy," Curr. Drug Saf. 3(1), 46-53 (2008), http://dx.doi.org/10.2174/157488608783333925.

4. F. Heitz, M. C. Morris, and G. Divita, "Twenty years of cell-penetrating peptides: from molecular mechanisms to therapeutics," Br. J. Pharmacol. 157(2), 195-206 (2009), http://dx.doi.org/10.1111/j.1476-5381.2009.00057.x.

5. E. Vivès, P. Brodin, and B. Lebleu, "Truncated HIV-1 Tat protein basic domain rapidly translocates through the plasma membrane," J. Biol. Chem. 272(25), 16010-16017 (1997), http://dx.doi.org/10.1074/jbc.272.25.16010.

6. A. M. Angeles-Boza et al., "Generation of endosomolytic reagents by branching of cellpenetrating peptides," Bioconjugate Chem. 21(12), 2164-2167 (2010), http://dx.doi.org/10 $.1021 / \mathrm{bc} 100130 \mathrm{r}$.

7. S. Galdiero et al., "Intracellular delivery: exploiting viral membranotropic peptides," Curr. Drug Metab. 13(1), 93-104 (2012), http://dx.doi.org/10.2174/138920012798356961.

8. P. L. Leopold et al., "Fluorescent virions: dynamic tracking of the pathway of adenoviral gene transfer vectors in living cells," Hum. Gene Ther. 9(3), 367-378 (1998), http://dx.doi .org/10.1089/hum.1998.9.3-367.

9. A. Falanga et al., "Membrane fusion and fission: enveloped viruses," Protein Pept. Lett. 16(7), 751-759 (2009), http://dx.doi.org/10.2174/092986609788681760.

10. S. Galdiero, M. Galdiero, and C. Pedone, "beta-Barrel membrane bacterial proteins: structure, function, assembly and interaction with lipids," Curr. Protein Pept. Sci. 8(1), 63-82 (2007), http://dx.doi.org/10.2174/138920307779941541.

11. S. Galdiero et al., "Fusogenic domains in herpes simplex virus type 1 glycoprotein H," J. Biol. Chem. 280(31), 28632-28643 (2005), http://dx.doi.org/10.1074/jbc.M505196200.

12. S. Galdiero et al., "Peptides containing membrane-interacting motifs inhibit herpes simplex virus type 1 infectivity," Peptides 29(9), 1461-1471 (2008), http://dx.doi.org/10.1016/j .peptides.2008.04.022. 
13. S. Galdiero et al., "The identification and characterization of fusogenic domains in herpes virus glycoprotein B molecules," Chembiochem 9(5), 758-767 (2008), http://dx.doi.org/10 $.1002 /($ ISSN)1439-7633.

14. S. Galdiero et al., "The presence of a single N-terminal histidine residue enhances the fusogenic properties of a membranotropic peptide derived from herpes simplex virus type 1 glycoprotein H," J. Biol. Chem. 285(22), 17123-17136 (2010), http://dx.doi.org/10 .1074/jbc.M110.114819.

15. S. Galdiero et al., "Role of membranotropic sequences from herpes simplex virus type I glycoproteins B and H in the fusion process," Biochim. Biophys. Acta 1798(3), 579-591 (2010), http://dx.doi.org/10.1016/j.bbamem.2010.01.006.

16. A. Falanga et al., "A peptide derived from herpes simplex virus type 1 glycoprotein $\mathrm{H}$ : membrane translocation and applications to the delivery of quantum dots," Nanomedicine 7(6), 925-934 (2011), http://dx.doi.org/10.1016/j.nano.2011.04.009.

17. Y. Tu and J. S. Kim, "A fusogenic segment of glycoprotein $\mathrm{H}$ from herpes simplex virus enhances transfection efficiency of cationic liposomes," J. Gene Med. 10(6), 646-654 (2008), http://dx.doi.org/10.1002/(ISSN)1521-2254.

18. R. Tarallo et al., "Clickable functionalization of liposomes with the gH625 peptide from Herpes simplex virus type I for intracellular drug delivery," Chemistry 17(45), 12659-12668 (2011), http://dx.doi.org/10.1002/chem.201101425.

19. D. Guarnieri et al., "Shuttle-mediated nanoparticle delivery to the blood brain barrier," Small (2012), http://dx.doi.org/10.1002/smll.201201870; PMID:23135878.

20. T. P. Carberry et al., "Dendrimer functionalization with a membrane-interacting domain of herpes simplex virus type 1: towards intracellular delivery," Chemistry 18(43), 13678-13685 (2012), http://dx.doi.org/10.1002/chem.201202358.

21. F. Pinaud et al., "Probing cellular events, one quantum dot at a time," Nat. Methods 7(4), 275-285 (2010), http://dx.doi.org/10.1038/nmeth.1444.

22. C. Kirchner et al., "Cytotoxicity of colloidal CdSe and CdSe/ZnS nanoparticles," Nano Lett. 5(2), 331-338 (2005), http://dx.doi.org/10.1021/n1047996m.

23. L. O. Cinteza, "Quantum dots in biochemical applications: advances and challenges," J. Nanophoton. 4(1), 42503-42505 (2010), http://dx.doi.org/10.1117/1.3500388.

24. J. Lovric et al., "Unmodified cadmium telluride quantum dots induce reactive oxygen species formation leading to multiple organelle damage and cell death," Chem. Biol. 12(11), 1227-1234 (2005), http://dx.doi.org/10.1016/j.chembiol.2005.09.008.

25. T. S. Hauck et al., "In vivo quantum-dot toxicity assessment," Small 6(1), 138-144 (2010), http://dx.doi.org/10.1002/smll.v6:1.

26. L. Ye et al., "A pilot study in non-human primates shows no adverse response to intravenous injection of quantum dots," Nat. Nanotechnol. 7(7), 453-458 (2012), http://dx.doi.org/10 $.1038 /$ nnano.2012.74.

27. H. Lee, I. K. Kim, and T. G. Park, "Intracellular trafficking and unpacking of siRNA/ quantum dot-PEI complexes modified with and without cell penetrating peptide: confocal and flow cytometric FRET analysis," Bioconjug. Chem. 21(2), 289-295 (2010), http://dx .doi.org/10.1021/bc900342p.

28. I. L. Medintz et al., "Intracellular delivery of quantum dot-protein cargos mediated by cell penetrating peptides," Bioconjug. Chem. 19(9), 1785-1795 (2008), http://dx.doi.org/10 $.1021 / \mathrm{bc} 800089 \mathrm{r}$.

29. J. B. Delehanty et al., "Spatiotemporal multicolor labeling of individual cells using peptidefunctionalized quantum dots and mixed delivery techniques," J. Am. Chem. Soc. 133(27), 10482-10489 (2011), http://dx.doi.org/10.1021/ja200555z.

30. E. Cukierman and D. R. Khan, "The benefits and challenges associated with the use of drug delivery systems in cancer therapy," Biochem.Pharmacol. 80(5), 762-770 (2010), http://dx .doi.org/10.1016/j.bcp.2010.04.020.

31. H. Maeda et al., "Tumor vascular permeability and the EPR effect in macromolecular therapeutics: a review," J. Contr. Release 65(1-2), 271-284 (2000), http://dx.doi.org/10.1016/ S0168-3659(99)00248-5. 
Falanga et al.: Review of a viral peptide nanosystem...

32. M. B. Bally et al., "Liposomes with entrapped doxorubicin exhibit extended blood residence times," Biochim. Biophys. Acta 1023(1), 133-139 (1990), http://dx.doi.org/10 .1016/0005-2736(90)90018-J.

33. S. Albrizio et al., "Driving forces in the delivery of penetratin conjugated G protein fragment," J. Med. Chem. 50(7), 1458-1464 (2007), http://dx.doi.org/10.1021/jm060935b.

34. G. R.Newkome and F. Vögtle, Eds., Dendrimers and Dendrons: Concepts, Synthesis Applications, Wiley-VCH, Weinheim (2001).

35. C. C. Lee et al., "Designing dendrimers for biological applications," Nat. Botechnol. 23(12), 1517-1526 (2005), http://dx.doi.org/10.1038/nbt1171.

36. S. Stevelmans et al., "Synthesis, characterization, and guest-host properties of inverted unimolecular dendritic micelles," J. Am. Chem. Soc. 118(31), 7398-7399 (1996), http:// dx.doi.org/10.1021/ja954207h.

37. M. Liu, K. Kono, and J. M. J. Fréchet, "Water-soluble dendritic unimolecular micelles: their potential as drug delivery agents," J. Control. Release 65(1-2), 121-131 (2000), http://dx .doi.org/10.1016/S0168-3659(99)00245-X.

38. C. Hawker and J. M. J. Fréchet, "A new convergent approach to monodisperse dendritic macromolecules," J. Chem. Soc. Chem. Commun. (15), 1010-1013 (1990), http://dx.doi .org/10.1039/c39900001010.

39. G. R. Newkome and C. D. Shreiner, "Poly(amidoamine), polypropylenimine, and related dendrimers and dendrons possessing different $1 \rightarrow 2$ branching motifs: an overview of the divergent procedures," Polymer 49(1), 1-173 (2008), http://dx.doi.org/10.1016/j.polymer .2007.10.021.

40. C. Ornelas and M. Weck, "Construction of well-defined multifunctional dendrimers using a trifunctional core," Chem. Commun. (38), 5710-5712 (2009), http://dx.doi.org/10.1039/ b913139f.

41. M. E. Fox, F. C. Szoka, and J. M. Frechet, "Soluble polymer carriers for the treatment of cancer: the importance of molecular architecture," Acc. Chem. Res. 42(8), 1141-1151 (2009), http://dx.doi.org/10.1021/ar900035f.

42. H. Kobayashi and M. W. Brechbiel, "Dendrimer-based nanosized MRI contrast agents," Curr. Pharm. Biotechnol. 5(6), 539-549 (2004), http://dx.doi.org/10.2174/1389201043376571.

43. J.-S. Zhang, F. Liu, and L. Huang, "Implications of pharmacokinetic behavior of lipoplex for its inflammatory toxicity," Adv. Drug Deliv. Rev. 57(5), 689-698 (2005), http://dx.doi .org/10.1016/j.addr.2004.12.004.

44. E. R. Gillies and J. M. Frechet, "Dendrimers and dendritic polymers in drug delivery," Drug Discov. Today 10(1), 35-43 (2005), http://dx.doi.org/10.1016/S1359-6446(04) 03276-3.

45. M. Najlah and A. D'Emanuele, "Crossing cellular barriers using dendrimer nanotechnologies," Curr. Opin. Pharmacol. 6(5), 522-527 (2006), http://dx.doi.org/10.1016/j.coph .2006.05.004.

46. F. P. Seib, A. T. Jones, and R. Duncan, "Comparison of the endocytic properties of linear and branched PEIs, and cationic PAMAM dendrimers in B16f10 melanoma cells," J. Contr. Release 117(3), 291-300 (2007), http://dx.doi.org/10.1016/j.jconrel.2006.10.020.

47. H. Kang et al., "Tat-conjugated PAMAM dendrimers as delivery agents for antisense and siRNA oligonucleotides," Pharm. Res. 22(12), 2099-2106 (2005), http://dx.doi.org/10 .1007/s11095-005-8330-5.

48. P. E. S. Smith et al., "Solid-state NMR reveals the hydrophobic-core location of poly(amidoamine) dendrimers in biomembranes," J. Am. Chem. Soc. 132(23), 8087-8097 (2010), http://dx.doi.org/10.1021/ja101524z.

49. S. Gunaseelan et al., "Surface modifications of nanocarriers for effective intracellular delivery of anti-HIV drugs," Adv. Drug Deliv. Rev. 62(4-5) 518-531 (2010), http://dx.doi.org/10 .1016/j.addr.2009.11.021.

50. J. C. Roberts, M. K. Bhalgat, and R. T. Zera, "Preliminary biological evaluation of polyamidoamine (PAMAM) Starburst dendrimers," J. Biomed. Mater. Res. 30(1), 53-65 (1996), http://dx.doi.org/10.1002/(ISSN)1097-4636.

51. C. Ornelas et al., "Construction of a well-defined multifunctional dendrimer for theranostics," Org. Lett. 13(5), 976-979 (2011), http://dx.doi.org/10.1021/ol103019z. 
52. E. Neuwelt et al., "Strategies to advance translational research into brain barriers," Lancet Neurol. 7(1), 84-96 (2008), http://dx.doi.org/10.1016/S1474-4422(07)70326-5.

53. G. Tosi et al., "Targeting the central nervous system: in vivo experiments with peptidederivatized nanoparticles loaded with Loperamide and Rhodamine-123," J. Contr. Release 122(1), 1-9 (2007), http://dx.doi.org/10.1016/j.jconrel.2007.05.022.

54. J. Kreuter, "Mechanism of polymeric nanoparticle-based drug transport across the bloodbrain barrier (BBB)," J. Microencapsul. 30(1), 49-54 (2013), http://dx.doi.org/10.3109/ 02652048.2012.692491.

55. S. V.Dhuria,L. R. Hanson, and W. H. Frey II, "Intranasal delivery to the central nervous system: mechanisms and experimental considerations," J. Pharm.Sci.99(4), 1654-1673(2010), http:// dx.doi.org/10.1002/jps.21924.

56. S. Azarmi, W. H. Roa, and R. Lobenberg, "Targeted delivery of nanoparticles for the treatment of lung diseases," Adv. Drug Deliv. Rev. 60(8), 863-875 (2008), http://dx.doi.org/10 .1016/j.addr.2007.11.006.

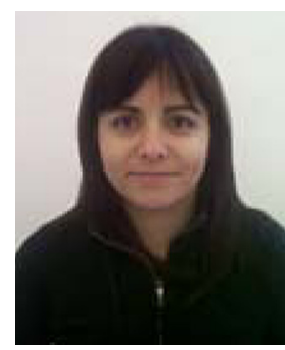

Annarita Falanga received her degree in food science and technology at the University of Naples Federico II in 2004. From 2004 to 2007, she took the $\mathrm{PhD}$ course in microbiological science and was involved in the synthesis and characterization of fusogenic domains derived from membrane glycoproteins of herpes symplex virus type I. From 2007 to 2012, she earned several research contracts at Consiglio Nazionale delle Ricerche, continuing her studies on peptides with antimicrobial activity. Her current research interests are in the development of stable formulation for radiopharmaceutical molecules.

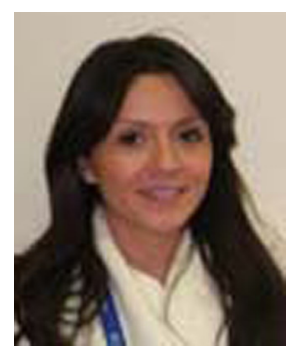

Rossella Tarallo received her degree in biotechnological science at the University of Naples Federico II in 2006. In 2009, she received a master's degree in medical biotechnology with a thesis on nanoparticle's internalization mechanisms. In 2010, she started the PhD course in industrial and molecular biotechnology. In 2011, she worked in the Department of Chemistry at New York University, USA, where she worked on the synthesis and characterization of peptidodendrimers as advanced nanosystems for biomedical applications. Her research projects concern the synthesis and characterization of novel biotechnological peptide systems for intracellular delivery of biomolecules such as liposomes, nanoparticles, and dendrimers functionalized with drugs for cancer therapy.

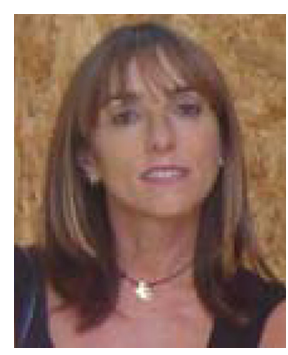

Emilia Galdiero obtained her MD degree from the University of Naples Federico II in 1988. She earned her specialization degree in hygiene and prevention medicine in 1992. She worked as a visiting scholar of infectious disease at the Istituto Superiore di Sanità in 1989. She was appointed a graduate technician in 1990 and worked until 1994 at the Microbiology Institute, Faculty of Pharmacy, University La Sapienza, Rome, and from 1995 to 2000 at the Department of General and Environmental Physiology, Hygiene, and Microbiology Section, University of Naples, Federico II. Since 2001, she has been faculty member in the Department of Structural and Functional Biology, University of Naples Federico II.

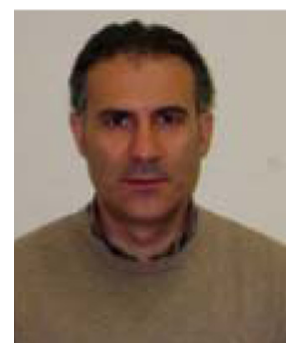

Marco Cantisani received his degree in chemistry at the University of Naples Federico II in 2001. From 2002 to 2005, he attended the PhD course in ambiental science at the University of Molise, Italy. During that time, he worked on antimicrobial peptides, in particular human beta-defensins. In 2009, he earned a researcher contract, and since then he has worked at the Centro Interdipartimentale di Ricerca sui Peptidi Bioattivi (CirpeB) in collaboration with CEINGE Scarl on antimicrobial peptide identification, synthesis, characterization, and biomedical applications for the treatment of high-social-impact diseases. 


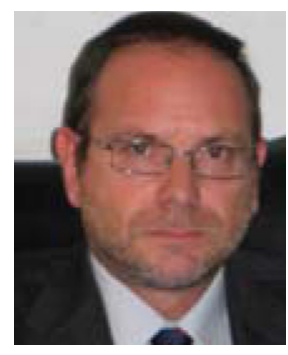

Massimiliano Galdiero obtained his MD degree from the University of Naples Federico II in 1992. He earned his $\mathrm{PhD}$ in virology from the University of Cambridge, UK, in 1998. He was appointed a lecturer in microbiology in 1994 at the Department of Animal Health of the Faculty of Veterinary Medicine, University of Naples Federico II. He moved to the Faculty of Medicine of the Second University of Naples, where he was appointed associate professor and then full professor of microbiology in 2004. His more recent research interests are focused on the study of host-microbial interactions and antimicrobial development.

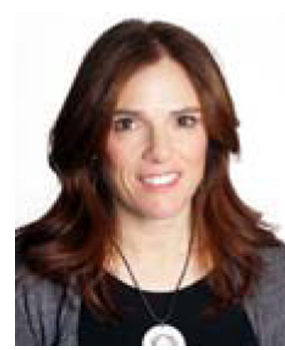

Stefania Galdiero completed her doctorate in chemistry in the field of peptide chemistry at the University of Naples Federico II in 1998. She carried out research activities at Columbia University, New York, from 1996 to 1997, working on the structure of alpha-hemolysin from S. aureus and phospholipid interactions. In 1999, she earned the position of lecturer in inorganic chemistry at the University of Naples, and since 2012, she has been adjunct professor at Loyola University, Chicago. She is a member of the Biostructures and Bioimages Institute of the National Research Council of Italy. Her recent research activities focus on the biological activity of bacterial porins and mechanisms of drug intracellular delivery. 